\title{
Drawing Simultaneously Embedded Graphs with Few Bends ${ }^{\star}$
}

\author{
Luca Grilli $^{1}$, Seok-Hee Hong ${ }^{2}$, Jan Kratochvíl ${ }^{3}$, and Ignaz Rutter ${ }^{3,4}$ \\ 1 Dipartimento di Ingegneria, Università degli Studi di Perugia \\ luca.grilli@unipg.it \\ 2 School of Information Technologies, University of Sydney \\ shhong@it.usyd.edu.au \\ 3 Department of Applied Mathematics, Faculty of Mathematics and Physics, \\ Charles University in Prague \\ honza@kam.mff.cuni.cz \\ 4 Institute of Theoretical Informatics, Karlsruhe Institute of Technology \\ rutter@kit.edu
}

\begin{abstract}
We study the problem of drawing simultaneously embedded graphs with few bends. We show that for any simultaneous embedding with fixed edges (SEFE) of two graphs, there exists a corresponding drawing realizing this embedding such that common edges are drawn as straight-line segments and each exclusive edge has a constant number of bends. If the common graph is biconnected and induced, a straight-line drawing exists. This yields the first efficient testing algorithm for simultaneous geometric embedding (SGE) for a non-trivial class of graphs.
\end{abstract}

\section{Introduction}

Let $G_{1}=\left(V_{1}, E_{1}\right)$ and $G_{2}=\left(V_{2}, E_{2}\right)$ be two graphs sharing a common graph $G=(V, E)=\left(V_{1} \cap V_{2}, E_{1} \cap E_{2}\right)$. The vertices and edges in $V_{i} \backslash V$ and $E_{i} \backslash E$ are called exclusive. The problem of finding a simultaneous drawing of $G_{1}$ and $G_{2}$ such that each graph is drawn in a planar way and the subdrawing of $G$ coincides in both drawings is a long-standing problem in Graph Drawing with applications to, e.g., dynamic graph drawing. The problem can be studied in a topological variant, SIMULTANEOUS EMBEDding WiTh FIXED EdGES (or SEFE for short), where edges are represented by arbitrary open Jordan curves between their endpoints or in the geometric variant, Simultaneous GEOMETRIC EMBEDDing (or SGE for short), where edges are represented by straight-line segments. Both problems naturally generalize to more than two input graphs. An important special case is the case of sunflower intersection, where one requires that the pairwise intersection of any two input graphs is the same.

\footnotetext{
* This work was started at the Bertinoro Workshop on Graph Drawing 2012. L. Grilli was partly supported by the MIUR project AMANDA "Algorithmics for MAssive and Networked DAta", prot. 2012C4E3KT_001. S. Hong was supported by ARC Future Fellowship and Humboldt Fellowship. Work by Jan Kratochvíl was supported by the grant no. 14-14179S of the Czech Science Foundation GAČR. Ignaz Rutter was supported by a fellowship within the PostdocProgram of the German Academic Exchange Service (DAAD).
}

C. Duncan and A. Symvonis (Eds.): GD 2014, LNCS 8871, pp. 40-51 2014.

(C) Springer-Verlag Berlin Heidelberg 2014 
The problem SGE is NP-hard [8] and, moreover, there are quite restricted graph classes that do not always admit an SGE, e.g., even a path and a tree do not always admit an SGE [3]. To date no efficient testing algorithms for a non-trivial class of restricted input instances is known.

In contrast, the complexity of testing the existence of a SEFE drawing for two input graphs is a long-standing open problem. Jünger and Schulz [13] showed that the problem is actually equivalent to determining planar embeddings of the two input graphs that induce the same embedding on the common graph. For three input graphs the problem is NP-complete [9]. In recent years considerable progress has been made, providing efficient testing algorithms for increasingly general sets of input instances. Most of the results revolve around assumptions on the connectivity or the maximum degree of the input graphs and the common subgraph. It is known that SEFE can be tested in polynomial time if the common subgraph has a fixed planar embedding [1], if the common graph is biconnected [2 10], if the two input graphs are biconnected and the common graph is connected or a forest [6], and if each connected component of the common graph is either biconnected or subcubic [18]. The last result can be improved to also allow connected components of the common graph that are outerplanar and whose cutvertices have degree at most 3 in the common graph [4]. See the recent survey by Bläsius et al. [5] for further details.

While the rephrasing of the original drawing problem SEFE as an embedding problem has certainly been fundamental in starting this evolution, it also comes at a disadvantage. Typically, the outputs of the above-mentioned algorithms are just planar embeddings of the input graphs, i.e., rotation systems and relative positions of the connected components, that coincide on the common graph. We call this a SEFE embedding. To obtain a visualization, it is necessary to transform this combinatorial description of a drawing into an actual drawing while preserving the given embedding. For clarity, we refer to such a drawing as a SEFE drawing and say that the SEFE drawing realizes the corresponding SEFE embedding. Although the complexity of SEFE is still open, the existing results allow efficient testing algorithms for a large range of instances, increasing the importance of the realization problem. The very first result on simultaneous drawings with few bends was obtained by Erten and Kobourov [7] in the context of SimultaneOUS EMBEDDing, where one only requires that common vertices are drawn the same, whereas shared edges may be drawn differently for different input graphs. They showed that three bends per edge suffice if the common graph does not contain any edges. Haeupler et al. [10] initiated the study of the realization problem for SEFE embeddings and showed that for any instance where the common graph is connected, it is always possible to find a SEFE drawing realizing a given SEFE embedding in such a way that one of the input graphs is drawn straight-line, whereas the other graph has at most as many bends per edge as the number of vertices in the common subgraph. We show that a constant number of bends per edge suffices if bends are allowed on the exclusive edges of both graphs, even if the common graph is disconnected.

A related notion is partially embedded graphs, where one seeks to extend a given drawing of a subgraph (partial drawing) into a planar drawing of the whole graph. Similar to the simultaneous drawing problem, the partial embedding problem has been studied both in the topological setting [1|12 18] and in the straight-line setting [15]17]. 
In fact, to obtain their result about SEFE realizations, Haeupler et al. [10] essentially show that $k$ bends per edge suffice when extending a given drawing with $k$ predrawn vertices provided that the predrawn graph is connected. Their result is then obtained by taking a straight-line drawing of $G_{1}$, considering the drawing it induces on the common graph $G$ and extending it to a drawing of $G_{2}$ with at most $|V|$ bends per edge, where $V$ is the vertex set of the common graph $G$. In the setting of partially embedded graphs their result is asymptotically tight; it is easy to construct an example that shows that $\Omega(k)$ bends per edge are necessary. To achieve our result, which only requires $O(1)$ bends on the exclusive edges, we allow bends on the exclusive edges of both graphs and make use of the fact that, in the SEFE realization problem, we can choose the drawing of the straight-line drawing of the common graph in such a way that it fits with both input graphs simultaneously.

Our Contribution. We study the problem of finding realizations of SEFE embeddings where the common graph is drawn without bends and the exclusive edges have few bends per edge. We refer to a drawing where the edges are represented by polygonal curves with at most $c_{1}$ bends per common edge and at most $c_{2}$ bends per exclusive edge as a $\left(c_{1}, c_{2}\right)$-drawing. In a SEFE drawing realizing a SEFE embedding, we require that the planar embeddings of the input graphs are preserved.

Our main result is that every SEFE embedding of two graphs admits a $(0, c)$-drawing with $c \leq 9$; see Section 4 . If the common graph is (bi)connected, we have $c \leq 3$ and this even holds for an arbitrary number of input graphs intersecting in a sunflower-way (i.e., the pairwise intersections of the input graphs are identical); see Section 3 . As a side result, we obtain the first efficient algorithm for testing SGE for a non-trivial class of graphs, namely for instances whose common graph is biconnected and an induced subgraph of the input graphs. Finally, we study lower bounds in Section 5 and show that some of the results from Section 3 are in fact tight. For the case of sunflower-intersection we show that there exist $k$-tuples of input graphs (with a disconnected common graph) that require $\Omega\left(\sqrt{2}^{k} / k\right)$ bends per edge. We note that all proofs are constructive and can be turned into efficient drawing algorithms.

\section{Preliminaries}

A graph $G=(V, E)$ is planar if and only if it can be drawn in the Euclidean plane such that its vertices are represented by points and its edges are represented by internally disjoint open Jordan curves between their endpoints. If $G$ is connected, a planar drawing can be combinatorially described by its rotation system, i.e., the circular ordering of the edges around each vertex and the choice of an outer face. We refer to this rotation system as the associated (combinatorial) embedding. For disconnected graphs, the embedding also encodes the relative positions of the connected components.

A plane graph $H$ is a planar graph with an associated planar embedding. A drawing of a plane graph is a planar drawing of the graph with its given planar embedding. A plane subgraph of $H$ is a subgraph $G$ of $H$ associated with the corresponding planar embedding induced by $H$. In this case we also say that $H$ is a plane supergraph of $G$. 
Consider a straight-line drawing $\Gamma$ of a planar graph $G$. A face $f$ is star-shaped if it contains a point $p$ such that the straight-line segment from $p$ to each vertex of $f$ lies inside $f$. The set of all such points $p$ is the kernel of the face. We say that $\Gamma$ is star-shaped if all its faces are star-shaped. We will frequently use the following lemma, stating that any planar graph admits a star-shaped drawing. Of course it is always possible to find a drawing where the vertices are in general position. This implies that the kernel of each face has positive area.

Lemma 1. Let $G=(V, E)$ be a plane graph. There exists a star-shaped planar straightline drawing of $G$ such that the kernel of the outer face contains points on or outside of the convex hull of the vertices in $G$.

Proof. To construct such a drawing, we create for each face $f$ of $G$ a new vertex $v_{f}$ that is connected to vertices incident to $f$ and embed it inside $f$. Afterwards, we triangulate the graph in such a way that the vertex $v_{0}$ added for the outer face $o$ is incident to the outer face. Call the resulting graph $G^{\star}$.

We then produce a straight-line drawing of $G^{\star}$. Since this drawing is planar and each vertex $v_{f}$ is connected by straight-line segments to all vertices incident to $f$, removing $v_{f}$ yields a drawing where face $f$ is star-shaped. We remove all vertices $v_{f}$ of all faces to obtain a star-shaped drawing. Note that the outer face $o$ of $G^{\star}$ is a triangle, and hence $v_{o}$ lies on or outside of the convex hull of $G$.

For an instance $G_{1}=\left(V_{1}, E_{1}\right)$ and $G_{2}=\left(V_{2}, E_{2}\right)$ of SEFE, we say that the common graph $G=(V, E)$ is induced if the induced subgraph of $V$ in $G_{1}$ and $G_{2}$ is $G$. This is equivalent to the statement that each exclusive edge has at least one endpoint that is not in $V$. Assuming that $G$ is induced will often simplify our arguments. Given a non-induced instance $\left(G_{1}, G_{2}\right)$ of SEFE together with a SEFE embedding, we can construct its associated induced instance by subdividing each exclusive edge once (with an exclusive vertex). Note that this operation does not change the common graph $G$. By interpreting the subdivision vertices as bends in a drawing of the associated induced instance, we obtain the following lemma.

Lemma 2. Let $\left(G_{1}, G_{2}\right)$ be an instance of SEFE with a fixed SEFE embedding and let $\left(G_{1}^{\prime}, G_{2}^{\prime}\right)$ be the associated induced instance. If $\left(G_{1}^{\prime}, G_{2}^{\prime}\right)$ admits a $(0, c)$-drawing, then $\left(G_{1}, G_{2}\right)$ admits a $(0,2 c+1)$-drawing.

Proof. Consider a $(0, c)$-drawing of $\left(G_{1}^{\prime}, G_{2}^{\prime}\right)$. We can interpret it as a SEFE drawing of $\left(G_{1}, G_{2}\right)$ by interpreting the subdivision vertices as bends. Consider a subdivided edge $e$. By assumption, each half-edge into which $e$ is subdivided has at most $c$ bends. Together with the additional bend at the subdivision vertex this amounts to a total of $2 c+1$ bends per edge.

\section{SEFE Drawing with (Bi-)Connected Common Graph}

In this section we study realizations of SEFE embeddings where the common graph is biconnected or connected. The main result of this section is the following theorem. 
Theorem 1. Let $\left(G_{1}, \ldots, G_{k}\right)$ be a sunflower instance of SEFE with pairwise common graph $G$ together with a SEFE embedding. Then the following SEFE drawings realizing the given SEFE embedding exist.

(i) If $G$ is biconnected and induced, there exists a (0,0)-drawing.

(ii) If $G$ is biconnected, there exists a $(0,1)$-drawing.

(iii) If $G$ is connected and induced, there exists a $(0,1)$-drawing.

(iv) If $G$ is connected, there exists a $(0,3)$-drawing.

Before we proceed to prove Theorem 1 we first mention an interesting implication; the first efficient algorithm for testing the existence of SGE on a non-trivial class of graphs. By Theorem 1 (i) two graphs whose common graph is biconnected and induced admit an SGE if and only if they admit a SEFE. The latter can be tested in linear time [2|10].

Corollary 1. There is a linear-time algorithm for SIMULTANEOUS GEOMETRIC EMBEDDING if the common graph is biconnected and induced.

The rest of this section is devoted to proving Theorem 1 . The main tool for the proof is the existence of certain planar straight-line drawings of the common graph, so-called universal drawings, that can be extended to a drawing of any plane supergraph of $G$ with few bends per edge. Theorem 1 is an immediate consequence of the existence of such drawings.

Let $G$ be a planar graph and let $\Gamma$ be a planar straight-line drawing of $G$. Let $H \supseteq G$ be a plane supergraph of $G$. We say that $\Gamma$ is $k$-extendable for $H$ if it can be extended to a drawing of $H$ that has at most $k$ bends per edge. The drawing $\Gamma$ is $k$-universal if it is $k$-extendable to every plane supergraph $H$. The drawing $\Gamma$ is induced $k$-universal if it is $k$-extendable for any plane supergraph $H$ that contains $G$ as an induced subgraph. Similar to Lemma 2] a drawing that is induced $k$-universal is $2 k+1$-universal.

Lemma 3. Let $G$ be a planar graph and let $\Gamma$ be an induced $k$-universal drawing of $G$. Then $\Gamma$ is $(2 k+1)$-universal.

Proof. Let $H \supseteq G$ be an arbitrary plane supergraph of $G$. Let $H^{\prime}$ be the graph obtained from $H$ by subdividing each edge of $H-G$ whose endpoints both belong to $H$. Since $\Gamma$ is induced $k$-universal, we find a drawing $\Gamma^{\prime}$ of $H^{\prime}$ extending $\Gamma$ that has at most $k$ bends per edge. By interpreting the subdivision vertices as bends, the drawing $\Gamma^{\prime}$ can be seen as a drawing of $H$ with at most $(2 k+1)$-bends per edge, extending $\Gamma$. This finishes the proof, since $H$ is an arbitrary plane supergraph of $G$.

We are now ready to prove the existence of universal drawings for biconnected planar graphs.

Theorem 2. Every biconnected plane graph has an induced 0-universal drawing.

Proof. Let $G$ be a biconnected plane graph. We claim that a star-shaped drawing of $G$, which exists by Lemma 1 is induced 0 -universal.

Let $H \supseteq G$ be a planar supergraph of $G$ with a fixed embedding that extends that of $G$. Without loss of generality, we assume that $H$ is a triangulation. If it is not, we add 
new vertices to triangulate its faces. Note that it may not be possible to triangulate $H$ without adding new vertices as this might violate the property of $G$ being an induced subgraph. All vertices and edges that are added during the triangulation can be removed after a drawing has been found.

The vertices and edges of $H-G$ are embedded in the faces of $G$. We show that, for each face $f$ of $G$, the parts of $H-G$ embedded in $f$ can be drawn inside $f$ without any bends. To this end, let $f$ be a face of $G$ and let $H^{\prime}$ denote the subgraph of $H$ consisting of the boundary of $f$ and all edges and vertices of $H$ that are embedded in $f$. Let further $C$ denote the facial cycle bounding $f$. Note that $C$ is a simple cycle since $G$ is biconnected. Then the graph $H^{\prime}$ is a planar graph where one face is bounded by $C$ and all remaining faces are triangles. Hence, considering $C$ as the boundary of the outer face of $H^{\prime}$, it follows that $H^{\prime}$ is internally triconnected and, moreover, it is triconnected if and only if it does not contain a chord of $C$. This is, however, not the case since $C$ is an induced subgraph of $H^{\prime}$. It hence follows that $H^{\prime}$ is triconnected. Thus, $H^{\prime}$ is triconnected and its outer vertices have been fixed to a star-shaped polygon with positive kernel. A result of Hong and Nagamochi [11, Theorem 10] shows that the given drawing of $C$ can be extended to one of $H^{\prime}$ without crossings and bends. Since this reasoning can be applied independently to all faces, we find the claimed extension.

For the outer face we use a technique similar to [10] and position the vertices of $H-G$ incident to $G$ on the boundary of a small disk in the interior of the kernel of the outer face such that the edges between $G$ and $H-G$ can be drawn in a planar way without intersecting the interior of the disk. Then, since the positioned exclusive vertices are in convex position, the remaining drawing can be completed without any bends by a Tutte drawing [19].

By applying Lemma3, we immediately obtain the following corollary.

Corollary 2. Every biconnected plane graph has a 1-universal drawing.

\section{Theorem 3. Every connected plane graph has an induced 1-universal drawing.}

Proof. Let $G$ be a connected planar graph with a fixed embedding. We show how to construct an induced 1-universal drawing of $G$. Let $v$ be a cutvertex of $G$, let $f$ be a face of $G$ on whose boundary $v$ occurs at least twice, and let $v u$ and $v w$ be two edges incident to $f$ and $v$ that are consecutive in the circular ordering around $v$. We call such a configuration an angle of $G$. We process this angle by adding to $G$ a new vertex $v^{\prime}$ with neighbors $u, v$ and $w$. We call $v^{\prime}$ the representative vertex of the angle; see Fig. 1, Call $G^{\prime}$ the graph resulting from $G$ after processing all angles in this way. Note that $G^{\prime}$ is biconnected and hence has an induced 0 -universal drawing $\Gamma^{\prime}$ by Theorem 2

We claim that the restriction $\Gamma$ of $\Gamma^{\prime}$ to $G$ is induced 1-universal. Let $H \supseteq G$ be a plane graph that contains $G$ as an induced subgraph. For each cutvertex $v$ of $G$, the incident edges of $H$ are embedded in one of the angles of $v$. Let $u$ and $w$ be the other two vertices of such an angle at $v$ and let $v v_{1}, \ldots, v v_{k}$ denote the edges of $H$ that are embedded inside this angle. We modify $H$ by creating a new vertex $v^{\prime}$ that is adjacent to $u, v$ and $w$. We further replace the edges $v v_{1}, \ldots, v v_{k}$ by $v^{\prime} v_{1}, \ldots, v^{\prime} v_{k}$, which is clearly possible in a planar way; see Fig. 1, where the edges of $E(H) \backslash E(G)$ are shown dashed. Let $H^{\prime}$ be the graph resulting from $H$ by treating all angles of $G$ in 


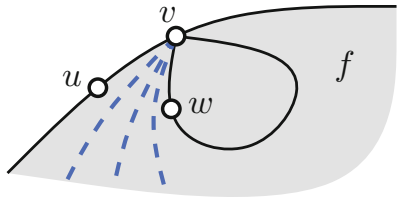

(a)

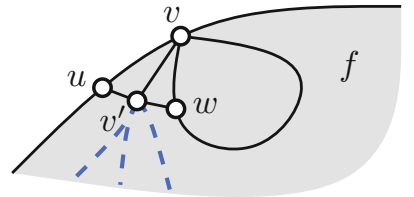

(b)

Fig. 1. Augmentation technique for removing cutvertices. (a) The graph $G$ is drawn solid, the exclusive edges of $H$ are dashed. (b) The modified graphs $G^{\prime}$ (solid) and $H^{\prime}$ (exclusive edges are dashed).

this way. Now $H^{\prime}$ contains $G^{\prime}$ as an induced subgraph. Since $\Gamma^{\prime}$ is induced 0 -universal, there exists a straight-line drawing of $H^{\prime}$ that extends $\Gamma^{\prime}$. To obtain a 1-bend drawing of $H$ that extends $\Gamma$, we remove from this drawing the edges $v^{\prime} u$ and $v^{\prime} w$ for each angle of $G$. Now each edge $x v$ of $H$, where $v$ is a cutvertex of $G$, is drawn with one bend at the position of the representative vertex of the corresponding angle. We can remove any overlaps of segments by slightly moving the bend points apart from each other without creating any crossings. Thus $\Gamma$ is induced 1 -universal.

Corollary 3. Every connected plane graph has a 3-universal drawing.

We note that Theorem 1 follows easily by applying one of Theorems 2 , 3 and Corollaries 2, 3 to the common graph of the SEFE embedding.

\section{SEFE Drawing for General Graphs}

Unfortunately, universal drawings cannot be used to prove the existence of SEFE drawings with few bends in general. Namely, Pach and Wenger [16] showed that drawing a planar graph with fixed vertex locations may require an edge with $\Theta(n)$ bends. Thus, even a graph consisting only of $n$ isolated vertices has no (induced) $o(n)$-universal drawing.

Our goal is to show that any SEFE embedding of two graphs $G_{1}$ and $G_{2}$ with common graph $G=G_{1} \cap G_{2}$ admits a $(0, c)$-drawing for some constant $c$. Unlike the previous constructions, this result does not generalize to an arbitrary number of graphs intersecting in a sunflower-fashion. In fact, we will see later, in Section 5, that for $k$ graphs $\Omega\left(\sqrt{2}^{k} / k\right)$ bends per edge are necessary even in the case of sunflowerintersection.

As a first step, we show how to construct a $(0,3)$-drawing of $\left(G_{1}, G_{2}\right)$ if the common graph is an induced subgraph of $G_{1}$ and $G_{2}$, respectively, and where we require that each connected component of the common graph is biconnected. Afterwards, we apply the technique from Theorem 3 to treat cutvertices in the connected components of $G$, resulting in $(0,4)$-drawings when $G$ is an induced subgraph. Then Lemma2 implies the existence of a $(0,9)$-drawing for any SEFE embedding. 


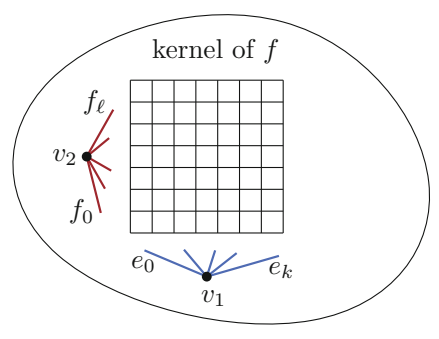

(a)

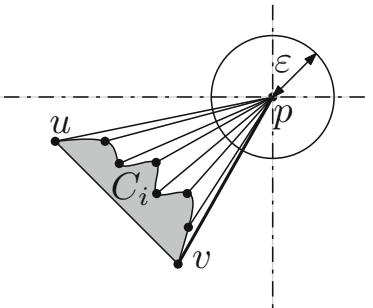

(b)

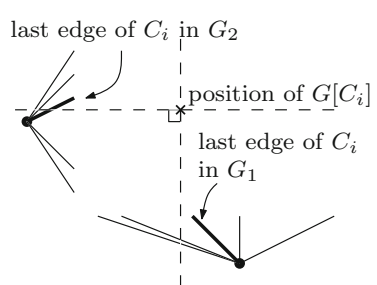

(c)

Fig. 2. Illustration of the placement of the $G\left[C_{i}\right]$ inside the face $f$ for a $(0,3)$-drawing

Theorem 4. Let $\left(G_{1}, G_{2}\right)$ be two planar graphs with a SEFE embedding. Assume further that $G=G_{1} \cap G_{2}$ is an induced subgraph of $G_{1}$ and $G_{2}$, respectively. If each connected component of $G$ is biconnected, there exists a $(0,3)$-drawing of $\left(G_{1}, G_{2}\right)$.

Sketch of Proof. Without loss of generality, we can assume that $G_{1}$ and $G_{2}$ are internally triangulated and that the outer face of $G$ does not contain any exclusive edges. Let $C$ be a connected component of $G$ and denote by $G[C]$ the subgraph of $G$ consisting of all vertices and edges that either belong to $C$ or are embedded inside some inner face of $C$. The graphs $G_{1}[C]$ and $G_{2}[C]$ are defined analogously. Note that if $C^{\prime}$ is a connected component of $G$ with $C^{\prime} \subseteq G[C]$, then $G\left[C^{\prime}\right] \subseteq G[C]$. Hence, the relation $C^{\prime} \prec C$ if and only if $G\left[C^{\prime}\right] \subseteq G[C]$ defines a partial ordering of the components of $G$ whose transitive reduction is a tree. We prove the following claim by induction on the depth of this tree; it implies the statement of the theorem.

Claim. Let $C$ be a connected component of $G$ with depth $d$. The induced SEFE of $G_{1}[C]$ and $G_{2}[C]$ admits a $(0,3)$-drawing such that the outer face of $G[C]$ is star-shaped.

The base case, where $G[C]=C$ is biconnected, follows from Theorem 11(i). For the induction step, assume that $C$ is a component such that $G[C]$ has depth $d$. The graph $C$ is biconnected, and we take a star-shaped drawing with positive kernel area in all faces. Clearly, the outer face is star-shaped. We show that we can embed the remaining parts of $G_{1}[C]$ and $G_{2}[C]$ inside the inner faces of $C$ using the given embedding and such that the result is a $(0,3)$-drawing. The faces of $C$ can be treated independently. In the following we fix an arbitrary internal face $f$ and denote by $C_{1}, \ldots, C_{c}$ the connected components of $G$ that are distinct from $C$ and incident to $f$. Note that $G\left[C_{i}\right]$ has depth at most $d-1$ and hence, by induction, we know that corresponding (0,3)-drawings of $G_{1}\left[C_{i}\right]$ and $G_{2}\left[C_{i}\right]$ exist for $i=1, \ldots, c$. We show how to arrange them in the interior of $f$ and how to draw the exclusive edges embedded inside $f$ to obtain a $(0,3)$ drawing of $G_{1}[C]$ and $G_{2}[C]$. We assume that the only exclusive edges of $G_{1}$ and $G_{2}$ are the ones embedded inside $f$. This is not a restriction since there is no interaction between exclusive edges in distinct faces of $G$. We can thus treat all faces independently.

Since $G_{1}$ and $G_{2}$ are triangulated, it follows that the subgraph induced by the exclusive vertices of $G_{i}$ that are embedded inside $f$ is connected, and we contract it into a single vertex $v_{i}$ for $i=1,2$, preserving the edge ordering of the given embedding. 


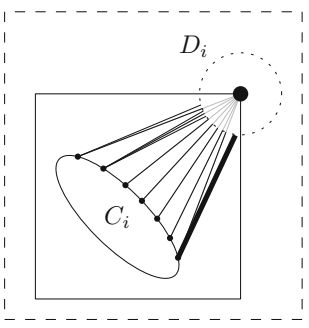

(a)

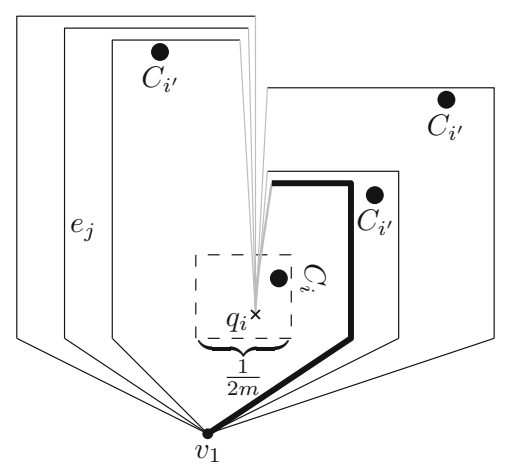

(b)

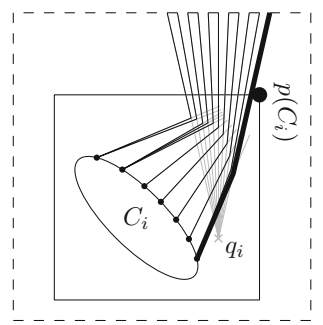

(c)

Fig. 3. Illustration of the drawing of exclusive internal edges

Note that we remove loops but not multiple edges. We will produce the largest part of the drawing inside a grid of size $2 \operatorname{deg}\left(v_{1}\right) \times 2 \operatorname{deg}\left(v_{2}\right)$, which we position inside the kernel of $f$. We distinguish the exclusive edges of $G_{1}$ and $G_{2}$ into two types. An exclusive edge is internal if its endpoint distinct from $v_{1}$ and $v_{2}$ belongs to one of the $C_{i}$ for $i=1, \ldots, c$. Otherwise the endpoint belongs to $C$ and the edge is external.

The vertices $v_{1}$ and $v_{2}$ are positioned below and left of the grid, respectively; see Fig. 2a By picking two external edges incident to $v_{1}$ and $v_{2}$ as the first edge, the circular ordering around these vertices determine corresponding linear orderings. Second, for each component $C_{i}$, we obtain a linear ordering of its incident internal edges by the ordering around a point in the kernel of the outer face; see Fig. 2b. Our goal is to draw the edges as shown in Fig. $3 \mathrm{~b}$. Since the linear ordering around $v_{1}$ and the linear ordering determined by $C_{i}$ do not necessarily coincide, this requires that component $C_{i}$ is positioned at a specific position, namely slightly left of the so-called last edge, which is shown bold in Fig. 3a and 3b In this way, the ordering of the edges around $v_{1}$ and $v_{2}$ imply a certain ordering of the components $C_{i}$. Since these orderings generally differ, we use the ordering around $v_{1}$ to determine the $x$-coordinate and the ordering around $v_{2}$ to determine the $y$-coordinate on the grid. Thus, each of the two vertices "sees" the components in the expected ordering; see Fig. 2c

We now sketch how to draw the edges of $G_{1}$. Graph $G_{2}$ is drawn analogously, but with exchanged $x$ - and $y$-coordinates, which corresponds to mirroring the drawing along a diagonal. Let $e_{0}, \ldots, e_{k}$ denote the internal edges incident to $v_{1}$ linearly ordered from left to right. Let $C_{i}$ be a component incident to edge $e_{j}$. We draw edge $e_{i}$ first from $v_{1}$ to the grid point $(2 j, 0)$, then vertically upwards up to some height $y\left(e_{j}\right)$, then horizontally above the position of $C_{i}$ (see Fig. 3b), and from there down to the position of $C_{i}$ and into its kernel. Finally, from there we route it to its endpoint in $C_{i}$ (see Fig. 3c). The main point is the height $y\left(e_{j}\right)$, which we choose such that $e_{j}$ passes above all components $C_{i^{\prime}}$ whose $x$-coordinate lies between the $x$-coordinate of $e_{j}$, i.e., $2 j$, and the $x$-coordinate of the target component $C_{i}$; see Fig. $3 \mathrm{~b}$. If $e_{j}$ is an internal edge, then we draw it from $v_{1}$ to $(2 j, 0)$ and from there vertically upwards through the hole grid. It is then not hard to reach the target vertex of $C$ with one or two more bends. 


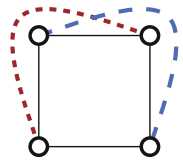

(a)

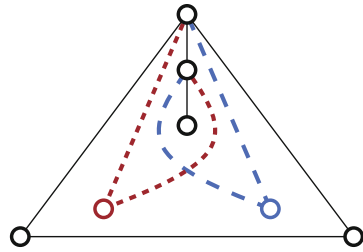

(b)

Fig. 4. SEFE instances that do not admit a $(0,0)$-drawing

Finally, we undo the contraction of $v_{1}$ and $v_{2}$ using a technique similar to Haeupler et al [10, Theorem 2].

If the connected components of the common graph are not biconnected, we use the same technique as in the proof of Theorem 3 to make them biconnected, showing that we obtain a $(0,4)$-drawing of the given SEFE embedding if the common graph is induced. Then, Lemma 2 implies the existence of a $(0,9)$-drawing for any SEFE embedding of two graphs.

Corollary 4. Let $\left(G_{1}, G_{2}\right)$ be two planar graphs whose common graph is induced with a SEFE embedding. Then $\left(G_{1}, G_{2}\right)$ admits a $(0,4)$-drawing.

Corollary 5. Any SEFE embedding of two graphs admits a (0, 9)-drawing.

\section{Lower Bounds}

In this section we study lower bounds on $c$ for $(0, c)$-drawings of SEFE embeddings. Since SEFE with an arbitrary number of input graphs is equivalent to the problem WEAK REALIZABILITY, which asks for a drawing of a graph specifying for each pair of edges whether they are allowed to cross, an example of Kratochvíl and Matoušek [14] shows that there are SEFE instances that require an exponential number of crossings between two edges. This implies that at least one of them must have an exponential number of bends. However, these graphs do not have sunflower-intersection.

We first show that the results from Theorem 11ii) and (iii) are tight; examples are given in Fig. 4. Afterwards, we prove that for a SEFE embedding of $k$ graphs with sunflower intersection $\Omega\left(\sqrt{2}^{k} / k\right)$ bends per edge are necessary.

Theorem 5. There exist SEFE embeddings that do not admit a (0,0)-drawing even when (i) the common graph is biconnected or (ii) the common graph is connected and induced.

Proof. For (i), consider a SEFE instance whose common graph is a cycle $C$ of length 4. The exclusive edges are the two chords of $C$, which belong to different graphs and are embedded outside of $C$; see Fig. 4a. Clearly, at least one of the exclusive edges requires a bend. 
For (ii), the common graph is a triangle with tip $u$ and a path of length 2 attached to $u$. Let the path be $u, v, w$. Additionally, each $G_{1}$ and $G_{2}$ contain one exclusive vertex that is adjacent to $u$ and $v$. The embedding is as shown in Fig. 4b. We claim that this SEFE does not admit a $(0,0)$-drawing.

Consider the red graph $G_{1}$. For a $(0,0)$-drawing, its exclusive vertex must be positioned such that it sees both $u$ and $v$. This can only be achieved if the angle at $v$ on the left side of the path $u v w$ is strictly greater than $\pi$. However, by applying the same arguments for $G_{2}$, we find that the angle at $v$ on the right side of the path $u v w$ must be strictly greater than $\pi$. This is obviously not possible simultaneously, and hence a $(0,0)$-drawing does not exist.

Theorem 6. There exist SEFE embeddings of $k$ graphs with sunflower intersection where any $(0, c)$-drawing has $c \in \Omega\left((\sqrt{2})^{k} / k\right)$.

Proof. We define a graph as follows. Let $C$ be a cycle of length four with vertices $N, E, S, W$ in this clockwise ordering. In the interior, we embed $2^{k}$ vertices, each labeled with a distinct binary vector of length $k$. Fix $k$ colors. We connect each vertex $v$ in the interior of $C$ by an edge of color $i$ to either $W$ or $E$. If the $i$ th bit of the binary vector associated with $v$ is 1 , the edge of color $i$ is $v W$, otherwise it is $v E$. Finally, we add edges of color $i$ from $S$ to $N$ for $i=1, \ldots, k$. We require that edges of the same color do not cross, whereas edges of different colors may cross and are drawn independently. We prove a lower bound on the number of bends in this model.

A SEFE instance can be obtained by interpreting each color as the exclusive edges of its own graph and subdividing each of the colored edges with an exclusive vertex of the corresponding color. In particular, the common graph consists of the cycle $C$ and the $2^{k}$ vertices in the interior and each $G_{i}$ additionally connects each of the $2^{k}$ vertices by a path of length 2 to either $E$ or $W$. The lower bound of the colored instance then also yields a lower bound for this SEFE instance.

Consider an arbitrary admissible drawing of the colored graph. Since each of the $2^{k}$ vertices is attached by an edge of color $i$ to either $W$ or $E$, each of the edges from $S$ to $N$ partitions the points inside $C$ into two sets of $2^{k-1}$ points. The $k S N$-edges then divide the interior of $C$ into $2^{k}$ areas, each nonempty, as it contains exactly one vertex.

Now consider only the $S N$-edges. Let $f \geq 2^{k}$ be the number of such areas in the interior of $C$, let $X$ be the number of crossings of the $S N$-edges in the drawing, and let $e$ be the number of arcs on the $S N$-edges in the arrangement. We thus have a planar graph with $f+1$ faces (including the outer face), $X+4$ vertices (including the vertices of $C$ ), and $e=2 X+k+4$ edges. By Euler's formula, we have $X+4-(2 X+k+4)+f+1=2$, i.e., $-X-k 2^{k}+1 \geq 2$ or equivalently $X \geq 2^{k}+1-k-1$. Since there are only $\left(\begin{array}{l}k \\ 2\end{array}\right)$ pairs of edges, at least one pair crosses $\frac{2\left(2^{k}+1-k-1\right)}{k(k-1)}=\Omega\left(2^{k} / k^{2}\right)$ times. Then at least one of them requires $\Omega\left((\sqrt{2})^{k} / k\right)$ bends.

\section{Conclusion}

We have studied the problem of constructing SEFE drawings with polygonal curves of low complexity. Our main result is that any SEFE embedding of two graphs can be 
drawn with at most nine bends per edge. Fewer bends suffice if the common graph is (bi)connected. Our main open questions concern lower bounds. What is the smallest $c_{0}$ such that every SEFE of two graphs admits a $\left(0, c_{0}\right)$-drawing? Is it possible to put some bends on the edges of $G$ in order to save bends on the exclusive edges?

\section{References}

1. Angelini, P., Di Battista, G., Frati, F., Jelínek, V., Kratochvíl, J., Patrignani, M., Rutter, I.: Testing planarity of partially embedded graphs. In: Discrete Algorithms (SODA 2010), pp. 202-221. SIAM (2010)

2. Angelini, P., Di Battista, G., Frati, F., Patrignani, M., Rutter, I.: Testing the simultaneous embeddability of two graphs whose intersection is a biconnected or a connected graph. J. Discrete Alg. 14, 150-172 (2012)

3. Angelini, P., Geyer, M., Kaufmann, M., Neuwirth, D.: On a tree and a path with no geometric simultaneous embedding. J. Graph Algorithms Appl. 16(1), 37-83 (2012)

4. Bläsius, T., Karrer, A., Rutter, I.: Simultaneous embedding: Edge orderings, relative positions, cutvertices. In: Wismath, S., Wolff, A. (eds.) GD 2013. LNCS, vol. 8242, pp. 220-231. Springer, Heidelberg (2013)

5. Bläsius, T., Kobourov, S.G., Rutter, I.: Simultaneous embedding of planar graphs. In: Tamassia, R. (ed.) Handbook of Graph Drawing and Visualization. CRC Press (2013)

6. Bläsius, T., Rutter, I.: Simultaneous PQ-ordering with applications to constrained embedding problems. In: Discrete Algorithms (SODA 2013), pp. 1030-1043. SIAM (2013)

7. Erten, C., Kobourov, S.G.: Simultaneous embedding of planar graphs with few bends. J. Graph Algorithms Appl. 9(3), 347-364 (2005)

8. Estrella-Balderrama, A., Gassner, E., Jünger, M., Percan, M., Schaefer, M., Schulz, M.: Simultaneous geometric graph embeddings. In: Hong, S.-H., Nishizeki, T., Quan, W. (eds.) GD 2007. LNCS, vol. 4875, pp. 280-290. Springer, Heidelberg (2008)

9. Gassner, E., Jünger, M., Percan, M., Schaefer, M., Schulz, M.: Simultaneous graph embeddings with fixed edges. In: Fomin, F.V. (ed.) WG 2006. LNCS, vol. 4271, pp. 325-335. Springer, Heidelberg (2006)

10. Haeupler, B., Jampani, K.R., Lubiw, A.: Testing simultaneous planarity when the common graph is 2-connected. J. Graph Algorithms Appl. 17(3), 147-171 (2013)

11. Hong, S.H., Nagamochi, H.: Convex drawings of graphs with non-convex boundary constraints. Discrete Appl. Math. 156(12), 2368-2380 (2008)

12. Jelínek, V., Kratochvíl, J., Rutter, I.: A kuratowski-type theorem for planarity of partially embedded graphs. Computational Geometry Theory \& Applications 46(4), 466-492 (2013)

13. Jünger, M., Schulz, M.: Intersection graphs in simultaneous embedding with fixed edges. J. Graph Algorithms Appl. 13(2), 205-218 (2009)

14. Kratochvíl, J., Matoušek, J.: String graphs requiring exponential representations. J. Comb. Theory, Ser. B 53(1), 1-4 (1991)

15. Mchedlidze, T., Nöllenburg, M., Rutter, I.: Drawing planar graphs with a prescribed inner face. In: Wismath, S., Wolff, A. (eds.) GD 2013. LNCS, vol. 8242, pp. 316-327. Springer, Heidelberg (2013)

16. Pach, J., Wenger, R.: Embedding planar graphs at fixed vertex locations. Graphs and Combinatorics 17, 717-728 (2001)

17. Patrignani, M.: On extending a partial straight-line drawing. International Journal of Foundations of Computer Science 17(5), 1061-1069 (2006)

18. Schaefer, M.: Toward a theory of planarity: Hanani-tutte and planarity variants. J. Graph Algorithms Appl. 17(4), 367-440 (2013)

19. Tutte, W.T.: How to draw a graph. London Math. Soc. s3-13(1), 743-767 (1963) 